\title{
Sympathetic nerve hyperactivity in non-diabetic offspring of patients with type 2 diabetes mellitus
}

\author{
R. J. Huggett • A. J. Hogarth • A. F. Mackintosh • \\ D. A. S. G. Mary
}

Received: 25 April 2006 / Accepted: 6 July 2006 / Published online: 13 September 2006

(C) Springer-Verlag 2006

\begin{abstract}
Aims/hypothesis Type 2 diabetes mellitus with hyperinsulinaemia is a state of sympathetic nerve hyperactivity, which can develop subsequently in non-diabetic first-degree offspring of patients with type 2 diabetes. Although both type 2 diabetes and sympathetic activation are major cardiovascular risk factors, the level of sympathetic nerve activity is as yet unknown in offspring of type 2 diabetic patients who are ostensibly normal. We therefore sought to quantify sympathetic nerve activity and its relationship to plasma insulin levels in ostensibly normal offspring of patients with type 2 diabetes, relative to a matched normal control group with no family history of type 2 diabetes.

Subjects and methods In two closely matched groups comprising 23 non-diabetic offspring of type 2 diabetic patients and 23 normal control individuals we measured resting muscle sympathetic nerve activity (MSNA) as the mean frequency of multi-unit bursts of MSNA and single units of MSNA (s-MSNA) with defined vasoconstrictor properties.

Results In offspring of type 2 diabetic patients, the fasting plasma levels of insulin $(7.4 \pm 0.80 \mu \mathrm{U} / \mathrm{ml})$ and s-MSNA $(45 \pm$ 3.2 impulses $/ 100$ beats $)$ were greater $(p<0.009$ and $p<0.003)$ than those in control persons $(4.6 \pm 0.76 \mu \mathrm{U} / \mathrm{ml}$ and $32 \pm 3.1$ impulses/100 beats, respectively). MSNA bursts and derived insulin resistance followed similar trends. Sympathetic nerve activity was significantly correlated to insulin levels $(p<0.0002)$ and resistance $(p<0.0001)$
\end{abstract}

R. J. Huggett • A. J. Hogarth $(\bowtie) \cdot$ A. F. Mackintosh •

D. A. S. G. Mary

Department of Cardiology, St. James's University Hospital,

Beckett Street,

Leeds LS9 7TF, UK

e-mail: andy_hogarth2003@yahoo.co.uk in offspring of type 2 diabetic patients, but not in control subjects.

Conclusions/interpretation Sympathetic activation occurred in normal non-diabetic offspring of patients with type 2 diabetes in proportion to their plasma insulin levels. Our data indicate the presence of a mechanistic link between hyperinsulinaemia and sympathetic activation, both of which could play a role in the subsequent development of cardiovascular risk factors.

Keywords Family history - Insulin .

Sympathetic nervous system - Type 2 diabetes mellitus

$\begin{array}{ll}\begin{array}{l}\text { Abbreviations } \\ \text { HOMA }\end{array} & \begin{array}{l}\text { homeostasis model assessment } \\ \text { homeostasis model assessment index } \\ \text { of insulin resistance } \\ \text { muscle sympathetic nerve activity }\end{array} \\ \text { MSNA } & \begin{array}{l}\text { single unit muscle sympathetic nerve } \\ \text { activity }\end{array}\end{array}$

\section{Introduction}

No information on the level of central sympathetic nerve output in ostensibly normal non-diabetic first-degree offspring of patients with type 2 diabetes mellitus has been published to date. It is reasonable to assume that sympathetic activation can occur in such subjects. For instance, offspring of type 2 diabetic patients are known to have raised plasma insulin levels and insulin resistance [1], which in turn can increase peripheral muscle sympathetic nerve activity (MSNA) as assessed by direct neural recording [2]. Also, offspring of type 2 diabetic patients 
may eventually develop type 2 diabetes mellitus [3, 4], which is associated with increased MSNA [5]. The issue of sympathetic activation is important because it has been linked to the pathogenesis of hypertension [6], a condition that commonly shares its presence and vascular complications with type 2 diabetes [5]. Indeed, accelerated progression towards developing cardiovascular risk factors and hypertension has been found in offspring of type 2 diabetic patients [4].

The present investigation was therefore designed to determine the magnitude of central sympathetic output and its relationship to plasma insulin levels in non-diabetic offspring of type 2 diabetic patients. For this purpose we directly measured the resting mean frequency of central sympathetic nerve output to the periphery, as well as plasma insulin levels, in two groups, namely non-diabetic offspring of type 2 diabetic patients, and closely matched control subjects with no family history of type 2 diabetes.

\section{Subjects and methods}

\section{Subjects}

Two groups of Caucasian subjects were investigated, the first comprising normal offspring of type 2 diabetic patients $(n=23)$, the second comprising normal control subjects $(n=23)$. The subjects of the two groups were matched for demographic data and haemodynamic variables. Only five members of the offspring group had two parents with type 2 diabetes. Both groups had similar occupational status and dietary habits, including a sodium intake of about $400 \mathrm{mmol} /$ day. Subjects were screened by history, physical and laboratory examination, and were excluded if evidence of conditions that may influence the autonomic nervous system was found, e.g. diabetes, cardiac dysrhythmias, vascular disease, microalbuminuria, neuropathy and existing or family history of hypertension. None of the subjects included had a fasting plasma glucose level greater than $5.6 \mathrm{mmol} / \mathrm{l}$. The investigation was conducted according to the principles of the Declaration of Helsinki (2000) of the World Medical Association and carried out with the approval of St. James's University Hospital Ethics Committee, with all subjects providing informed written consent.

Waist circumference was measured as minimal circumference at the navel and hip circumference as the widest circumference at the hips and buttocks; WHR was used as an index of regional adipose tissue distribution. Plasma insulin and glucose were measured through a venous sample, as previously described [5,7]. For insulin, the coefficient of variation of within-assay measurements ranged from 2.1 to $3.7 \%$, and that between assays ranged from 3.3 to $3.8 \%$. For glucose, the coefficient of variation of within-assay measurement was $0.9 \%[5,7]$. Insulin resistance was assessed by the homeostasis model assessment (HOMA), whereby the HOMA index of insulin resistance $($ HOMA-IR $)=$ fasting glucose $(\mathrm{mmol} / \mathrm{l}) \times$ (fasting insulin $[\mu \mathrm{U} / \mathrm{ml}] / 22.5$ ).

General protocol

The details of the protocol used in every subject have been previously published $[5,7]$. Briefly, all investigations were performed in the semi-supine position under similar conditions whilst avoiding confounding factors that can influence MSNA [5-7]. Changes in heart rate and arterial pressure were monitored and recorded using a standard ECG and a Finometer device (FMS, Arnhem, the Netherlands). Blood flow to the muscle of the left calf was obtained using standard mercury in silastic strain-gauge plethysmography (Hokanson, Bellevue, WA, USA). The details of recording and quantifying the mean frequency of post-ganglionic MSNA from the right peroneal nerve have been published previously [5-7]. Briefly, these include the mean frequency of either single units of MSNA (s-MSNA) or the integrated and rectified bursts of multi-units. Both had vasoconstrictor properties, and their frequency was expressed in terms of impulses or bursts per minute and per 100 cardiac beats.

\section{Statistics}

Differences between the two groups were assessed by Student $t$-tests for unpaired variables, as data of both groups were normally distributed. The least-squares technique was used to assess the linear relationship between variables. We considered $p$ values of less than 0.05 to be statistically significant. Data are presented as mean \pm SEM.

\section{Results}

The details of the two study groups are shown in Table 1 . The groups were matched for age, body weight, BMI, WHR, arterial pressure indices, heart rate and sex $\left(\chi^{2}=0.095 ; p>0.30\right)$.

Group analysis showed that all measures of the mean frequency of sympathetic nerve activity of s-MSNA and MSNA bursts per minute and per 100 beats were significantly greater in offspring of type 2 diabetic patients than in the control group (Table 1, Fig. 1). In the former, the s-MSNA and MSNA burst hyperactivity amounted to an increase of about 40 and $32 \%$, respectively, of the values found in control subjects. The ratio of the frequency of s-MSNA to MSNA bursts was significantly greater $(p<0.03)$ 
Table 1 Data of the two groups, non-diabetic offspring of type 2 diabetic patients and normal control persons with no family history of type 2 diabetes

\begin{tabular}{llll}
\hline Variable & $\begin{array}{l}\text { Offspring } \\
\text { group }\end{array}$ & $\begin{array}{l}\text { Control } \\
\text { group }\end{array}$ & $\begin{array}{l}p \\
\text { values }\end{array}$ \\
\hline Number (female) & $23(16)$ & $23(14)$ & 0.3788 \\
Age (years) & $37 \pm 2.1$ & $37 \pm 2.5$ & 0.4466 \\
BMI (kg/m ${ }^{2}$ ) & $29 \pm 1.0$ & $29 \pm 1.1$ & 0.4441 \\
Weight (kg) & $79 \pm 2.9$ & $81 \pm 3.2$ & 0.2040 \\
WHR & $0.87 \pm 0.016$ & $0.85 \pm 0.019$ & 0.2715 \\
Heart rate (beats/minute) & $66 \pm 2.0$ & $66 \pm 2.4$ & 0.4782 \\
Mean ABP (mmHg) & $91 \pm 1.6$ & $91 \pm 1.5$ & 0.4760 \\
Systolic ABP (mmHg) & $123 \pm 2.3$ & $121 \pm 2.0$ & 0.1747 \\
Diastolic ABP (mmHg) & $75 \pm 1.3$ & $77 \pm 1.3$ & 0.1750 \\
Fasting glucose (mmol/l) & $5.1 \pm 0.10$ & $4.8 \pm 0.11$ & 0.0640 \\
Fasting insulin ( $\mu \mathrm{U} / \mathrm{ml})$ & $7.4 \pm 0.80$ & $4.6 \pm 0.76$ & 0.0088 \\
HOMA-IR (U) & $1.7 \pm 0.20$ & $1.0 \pm 0.19$ & 0.0107 \\
s-MSNA (impulses/100 & $45 \pm 3.2$ & $32 \pm 3.1$ & 0.0023 \\
beats) & & & \\
s-MSNA (impulses/min) & $30 \pm 1.9$ & $20 \pm 1.8$ & 0.0002 \\
MSNA bursts (bursts/100 & $37 \pm 2.9$ & $28 \pm 3.0$ & 0.0229 \\
beats) & & & \\
MSNA bursts & $24 \pm 1.7$ & $18 \pm 1.7$ & 0.0065 \\
(bursts/min) & & & \\
\hline
\end{tabular}

Data are mean \pm SEM.

$A B P$ Arterial blood pressure, MSNA multi-unit muscle sympathetic nerve activity, $s-M S N A$ single unit muscle sympathetic nerve activity are expressed per 100 cardiac beats and per minute. $p$ values refer to unpaired $t$-tests

in offspring of type 2 diabetic patients $(1.26 \pm 0.035)$ than in control subjects $(1.15 \pm 0.028)$. Comparison of the offspring with the control group also showed that fasting plasma insulin and HOMA-IR were greater in the former group (Table 1).

Within-subject analysis showed, as expected from previously reported findings $[5,7]$, that the measures of sympathetic activity were not related to the level of arterial pressure indices or BMI $(0.01<\Gamma<0.31$; at least $p>0.05)$, but showed a significant correlation to age $(0.80<\Gamma<0.90$; at least $p<0.001)$ in each of the two groups. However, the measures of sympathetic nerve activity were positively correlated to the levels of plasma insulin $(0.60<\Gamma<0.75$; at least $p<0.0001)$ and HOMA-IR $(0.70<\Gamma<0.77$; at least $p<0.0001)$ in the offspring group, but not in the control group $(0.10<\Gamma<0.22$, at least $p>0.18$, and $0.10<\Gamma<0.20$, at least $p>0.16$, respectively).

\section{Discussion}

We have shown for the first time that non-diabetic and nonhypertensive offspring of patients with type 2 diabetes mellitus have increased central sympathetic nerve activity

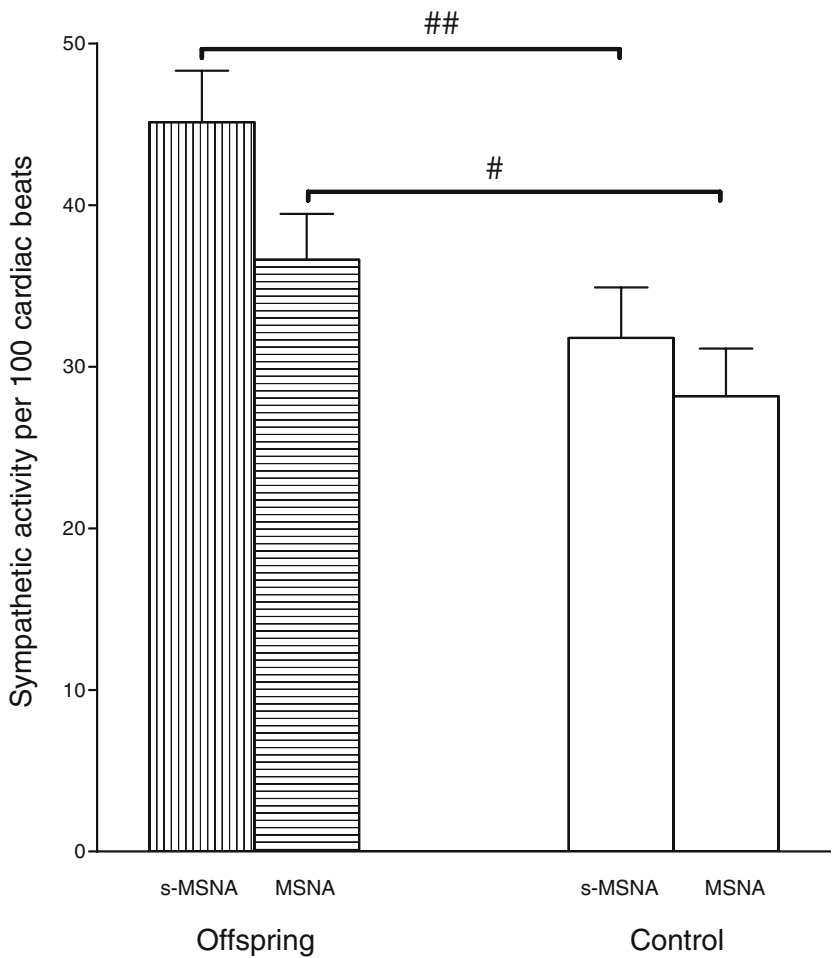

Fig. 1 The resting mean frequency of single unit muscle sympathetic nerve activity ( $s-M S N A)$ and that of multi-unit bursts of MSNA in non-diabetic offspring of type 2 diabetic patients and in normal control subjects with no family history of type 2 diabetes. Values are expressed as mean (height of columns) and SEM (bars). ${ }^{\#} p<0.04$ and ${ }^{\#} p<0.005$ for between-group comparisons (unpaired Student $t$-test)

to the periphery. In addition, this sympathetic hyperactivity was found to occur in proportion to the increase in levels of plasma insulin and insulin resistance. Our findings also confirm previous reports showing that offspring of type 2 diabetic patients may have raised insulin levels and insulin resistance [1]. Our findings suggest that the mechanisms involved in the central sympathetic activation include raised insulin levels.

In the present investigation, we avoided the confounding effects of race, age, body weight, time of day, dietary sodium intake, large meal and attendant visceral distension, alcohol, nicotine and exercise on sympathetic nerve activity as previously described [5-7]. Individuals from both groups were matched for demographic and haemodynamic variables, although this required control subjects to be of the same increased body weight as members of the offspring group. However, we have previously shown that such normal overweight subjects with no hormonal abnormality have normal levels of MSNA [7], and that BMI was not related to sympathetic nerve activity $[5,7]$ as in the present investigation. It is therefore likely that the raised MSNA and insulin data in the offspring group were not caused by these confounding factors. In addition, we found that MSNA was positively correlated to fasting plasma insulin 
and insulin resistance in the offspring group at a time when this was not found in control subjects, who had normal levels of plasma insulin and sympathetic activity. Although such a correlation does not establish a cause and effect, our findings suggest that MSNA hyperactivity in the offspring group was related to the raised levels of plasma insulin and insulin resistance.

On the basis of our findings, two opposing mechanisms involving sympathetic activation and the insulin resistance found in the offspring group are conceivable. First, offspring of type 2 diabetic subjects could have developed insulin resistance through sympathetic activation. This, however, is more likely to occur in pathological conditions such as diabetes and hypertension [5-7]. Second, there is evidence in normal humans and experimental animals $[2,8]$ that insulinaemia activates the central sympathetic drive. Indeed the nature of the sympathetic hyperactivity, of a greater s-MSNA:MSNA burst ratio in offspring of type 2 diabetic patients, in the present investigation was consistent with an increase in central sympathetic nerve output. As previously reported, s-MSNA represents the mean frequency of efferent sympathetic nerve activity and its ratio to MSNA bursts in the same recordings reflects central activation [6]. These considerations make it possible that raised plasma insulin level and insulin resistance could lead to the sympathetic activation observed in normal offspring of type 2 diabetic patients.

The present findings also have implications regarding potential health risks for persons who are ostensibly healthy offspring of type 2 diabetic patients. Such risks include the development of metabolic abnormalities, hypertension and coronary heart disease [4]. It could be argued that the sympathetic hyperactivity seen in the offspring group could be a factor in the development of these risk factors. Although we did not investigate this aspect and examine all indicators of cardiovascular risk, previous reports have linked sympathetic activation to the progression of insulin resistance [9, 10], hypertension and its complications [6]. While cardiovascular disease may arise through complex interactions between these factors, our findings suggest that sympathetic activation plays an important role.

Acknowledgements The authors would like to thank The Hypertension Trust and St James's Hospital Cardiology Research Fund for sponsoring this work. We also thank J. Bannister and J. Corrigan for technical assistance.

\section{References}

1. Vaag A, Lehtovirta M, Thye-Ronn P, Groop L, European Group of Insulin Resistance (2001). Metabolic impact of a family history of type 2 diabetes. Results from a European Multicentre Study (EGIR) Diabet Med 18:533-540

2. Scherrer U, Sartori C (1997) Insulin as a vascular and sympathoexcitatory hormone. Implications for blood pressure regulation, insulin sensitivity, and cardiovascular morbidity. Circulation 96:4104-4114

3. Meigs JB, Cupples LA, Wilson PW (2000) Parental transmission of type 2 diabetes: the Framingham Offspring Study. Diabetes 49:2201-2207

4. Srinivasan SR, Frontini MG, Berenson GS (2003) Longitudinal changes in risk variables of insulin resistance syndrome from childhood to young adulthood in offspring of parents with type 2 diabetes: the Bogalusa Heart Study. Metabolism 52:443-450

5. Huggett RJ, Scott EM, Gilbey SG, Stoker JB, Mackintosh AF, Mary DASG (2003) Impact of type 2 diabetes mellitus on sympathetic neural mechanisms in hypertension. Circulation 108:3097-3101

6. Mary DASG, Stoker JB (2003) The activity of single vasoconstrictor nerve units in hypertension. Acta Physiol Scandinav 177:367-376

7. Huggett RJ, Scott EM, Gilbey SG, Bannister J, Mackintosh AF, Mary DASG (2005) Disparity of autonomic control in type 2 diabetes mellitus. Diabetologia 48:172-179

8. Muntzel MS, Anderson EA, Johnson AK, Mark AL (1995) Mechanisms of insulin action on sympathetic nerve activity. Clin Exp Hypertens 17:39-50

9. Daly PA, Landsberg L (1991) Hypertension in obesity and NIDDM. Role of insulin and sympathetic nervous system. Diabetes Care 14:240-248

10. Lembo G, Vecchione C, Iaccarino G, Trimarco B (1996) The crosstalk between insulin and the sympathetic nervous system: possible implications in the pathogenesis of essential hypertension. Blood Press 1(Suppl):38-42 